\title{
Multidisciplinary approach for management of traumatic subgingival crown-root fractured central incisor: A case report
}

\author{
Kishan Agarwal $^{1 *}$, Praveen Singh Samant ${ }^{2}$, Shreya $^{3}$, Nikhil Ashok ${ }^{4}$, Ritwik Shyamal $^{5}$ \\ ${ }^{1,3-5} \mathrm{MDS},{ }^{2}$ Professor and Head of Department, ${ }^{1-3,5}$ Dept. of Conservative Dentistry and Endodontics, ${ }^{4}$ Dept. of Orthodontics and \\ Dentofacial Orthopedics, ${ }^{1-5}$ Saraswati Dental College, Lucknow, Uttar Pradesh, India \\ *Corresponding Author: Kishan Agarwal \\ Email: kishan25021991@gmail.com
}

\begin{abstract}
This case report describes a multidisciplinary approach to treat subgingivally fractured permanent right maxillary central incisor using orthodontic forced eruption to facilitate prosthetic restoration. A 23-year-old female patient reported at the Department of Conservative Dentistry and Endodontics with chief complaint of a fractured tooth and its unaesthetic appearance. Intraoral examination revealed that the maxillary right central incisor had sustained a oblique crown root fracture with pulp exposure. The treatment included endodontic procedure and orthodontic root extrusion using MBT mini brackets of .022 slots with ligation of .012 nickel titanium wire before going to prosthetic rehabilitation. Approximately $4.00 \mathrm{~mm}$ of extrusion of the tooth was obtained within 21 days with $0.5 \mathrm{~mm}$ extrusion of fracture margin supragingivally. A fiber post cementation, use of diode laser to correct the gingival margin and final restoration was performed with a porcelain fused to metal crown.
\end{abstract}

Keywords: Crown-Root fracture, Multidisciplinary management, Root canal treatment.

\section{Introduction}

Recently the incidences of traumatic injuries to the anterior teeth have been increased as an outcome of leisure activities, where the crown fractures are most common. These traumatic dental injuries may result in simple trauma by minimally damaging enamel or become complex by extending below the cemento-enamel junction and involve enamel, dentine, and cementum, may or may not involve the pulp. Crown-root fractures are very common but due to the complexity of the injury, these are frequently presented with the treatment problems. ${ }^{1}$ Dental trauma affects the maxillary central incisors in $80 \%$ to $83 \%$ of the patients. In young patients, trauma in permanent tooth could be a considerable owing to functional and esthetic causes. ${ }^{2-5}$ Diagnosis of the severity of trauma is made by clinical examination, and radiographic examination. ${ }^{6}$

Along with fracture types, here come the difficulties of the restorations which depend on the type of fracture. According to the complexity, the type of occlusion, prognosis and the Dean's Classification ${ }^{7}$, oblique fractures (type B according to Dean's classification) are found more difficult to be treated than any other fractures. Due to increased concern towards esthetics, patients often wish to recover their smile immediately at the very first appointment and demand its maintenance throughout subsequent treatment procedures. $^{8}$

The treatment strategy is determined by the lag time esthetic requirements. The treatment options that should be discussed includes:-

1. maintenance and use of the fractured portion either as a temporary or permanent crown,

2. definitive crown after an orthodontic extrusion or a crown lengthening,

3. or extraction of the residual tooth followed by an immediate or delayed implant surgery. ${ }^{8}$

Further, treatment plan of such complicated fractures is challenging and to achieve success a wise consideration of biological, esthetic, functional and economic aspects is an important criteria. Literature comes up with the various treatment strategies for crown-root fractures involving fragment removal and restoration; gingivectomy and osteotomy (crown lengthening); orthodontic extrusion with/without gingivoplasty; surgical extrusion; vital root submergence; and extraction followed by surgical implants or fixed partial dentures.

The ultimate treatment plan depends on the various factors regarding location, extent and nature of fracture as well as the morphology of the injured tooth. ${ }^{9}$

Orthodontic extrusion ${ }^{10}$ is a conservative procedure for raising the fracture line above the epithelial margins by elevating the sound tooth structure within the socket that makes it easier to prepare the proper finishing margins. ${ }^{11}$

This case report describes a multidisciplinary approach using orthodontic extrusion, followed by gingivectomy to facilitate the endodontic restoration of a subgingivally fractured maxillary permanent central incisor.

\section{Case Report}

A 23 year old female patient reported in the Department of Conservative Dentistry and Endodontics, Saraswati Dental College, Lucknow, with the chief complaint of broken tooth in her upper front tooth region since ten days. She gave the history of trauma (falling from height), ten days back and a sharp shooting pain at the time of trauma followed by mild continuous pain which subsided eventually after seven days. The patient's personal, medical and family histories were found to be non-contributory, and the results of the extraoral examination were also unremarkable.

\section{Intra oral examination}

The intraoral clinical examination revealed a complicated oblique crown root fracture involving distal aspect of the maxillary right permanent central incisor (Fig. 1) which was extending subgingivally from the palatal aspect. (Fig. 2) As 
fractured segment was lost during trauma, radiographic assessment was done to confirm the extent of fracture and remaining root length. Radiograph examination showed that the root had sufficient length, width and matured apex without any fracture, to be considered for extrusion (Fig. 3) and also, it was associated with periapical radiolucency and periodontal widening.

\section{Treatment aims}

These were as follows:-

1. Root canal treatment of the central incisor.

2. Intra-canal medicament.

3. Orthodontic extrusion.

4. Crown lengthening (gingivectomy) with diode laser.

5. Fiber post and core build-up and crown prosthesis to restore the esthetics.

\section{Treatment plan}

Orthodontic extrusion was considered as the best choice among all the other treatment options for extrusion in such cases.

The treatment plan consisted of multidisciplinary approach for plaque control, conservative and endodontic treatment, orthodontic extrusion, laser assisted periodontal surgery and prosthodontic restoration.

\section{Treatment Progress}

In the first appointment, conservative access cavity was prepared, pulp was extirpated and a working length radiograph was taken (Fig. 4). Cleaning and shaping was done in the same appointment followed by calcium hydroxide dressing (Fig. 5). Further orthodontic treatment was started, which consisted of forced eruption of tooth \#11using MBT mini brackets of .022 slots and ligation of .012 nickel titanium wire. Extent of fracture was estimated and $4 \mathrm{~mm}$ of extrusion was considered sufficient for the adequate ferrule preparation during final restoration, keeping in mind that crown and root ratio should not be less than $1: 1$, so that the sufficient biological width could be maintained.

After 4 weeks, $4 \mathrm{~mm}$ of desired extrusion was observed (Fig. 6,7) leading to $0.5-1 \mathrm{~mm}$ supragingival exposure of fracture margin, which was confirmed radiographically by observing the apex of central incisor which had drifted incisally in the socket (Fig. 9). Further arch stabilization was done for 8 weeks using 17 x 25 stainless steel wire. Finally, after desirable exposure of the fracture margin till gingival level (Fig. 8), the orthodontic brackets were removed after almost three months. Calcium hydroxide was removed from the canal and obturation was done by cold lateral condensation technique which was followed by fiber post and core build-up (Fig.10). Clinically gingival margin of the restored central incisor was found to be unpleasant (Fig. 11). Crown lengthening was done using diode laser to re-contour the gingival margin in relation to the adjacent teeth (Fig. 12, 13). Finally metal ceramic prosthesis was cemented to restore the esthetic need of the patient (Fig. 14).

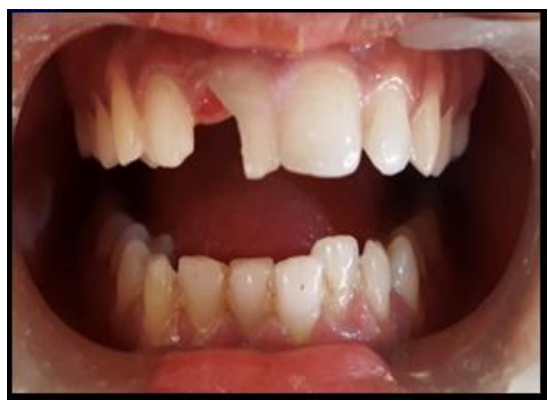

Fig. 1: Clinical picture showing complicated crown fracture in \#11;

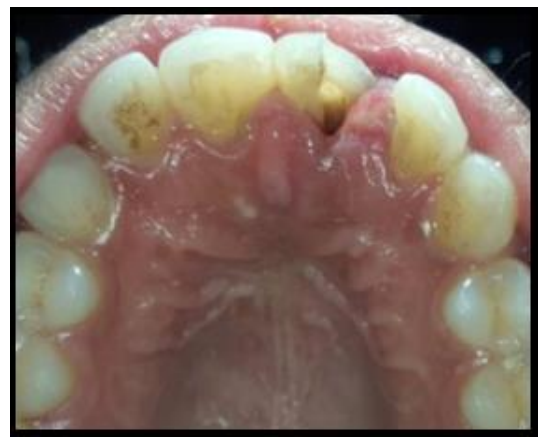

Fig. 2:Clinical picture showing palatal aspect of fractured tooth in \#11:

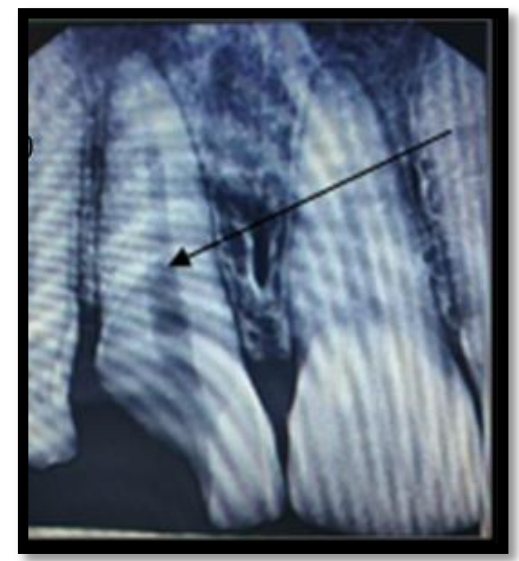

Fig. 3: Radiographic image showing fracture line (arrow) extending till coronal third of the tooth \#11;

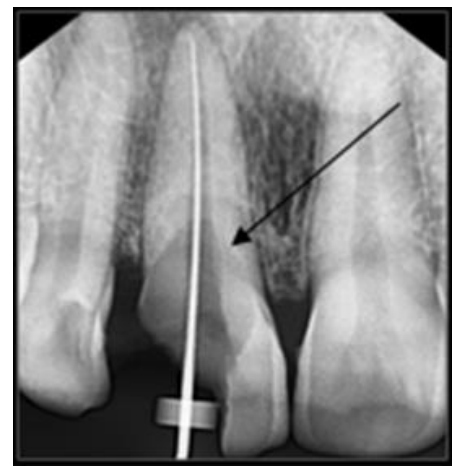

Fig. 4: Radiographic working length determination of tooth $\# 11$; fracture line is shown with an arrow; 


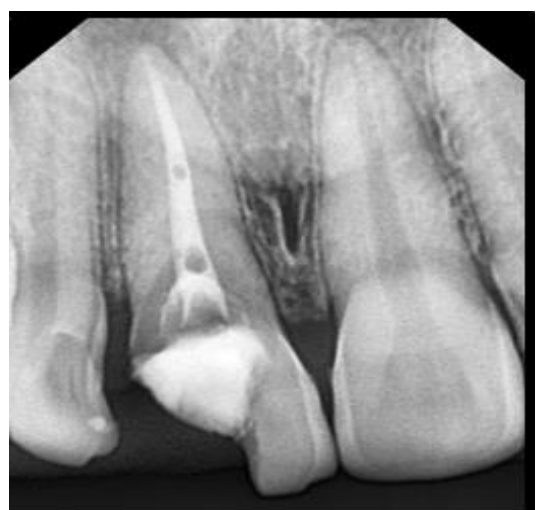

Fig. 5: Calcium hydroxide intracanal medicament in tooth \#11;

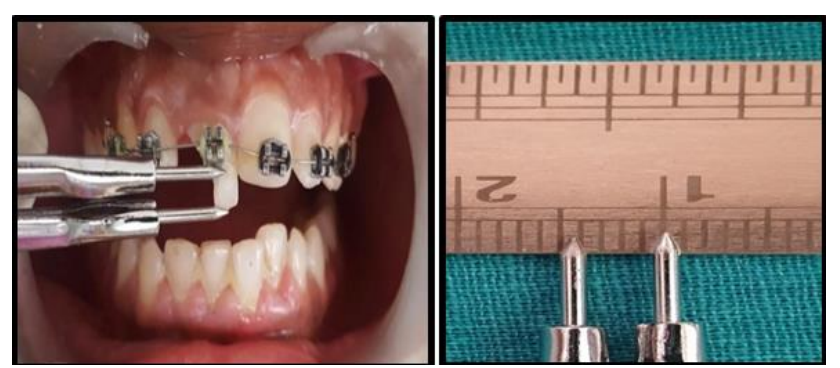

Fig. 6,7: Clinical evidence of increased crown length after forced orthodontic extrusion of tooth \#11;

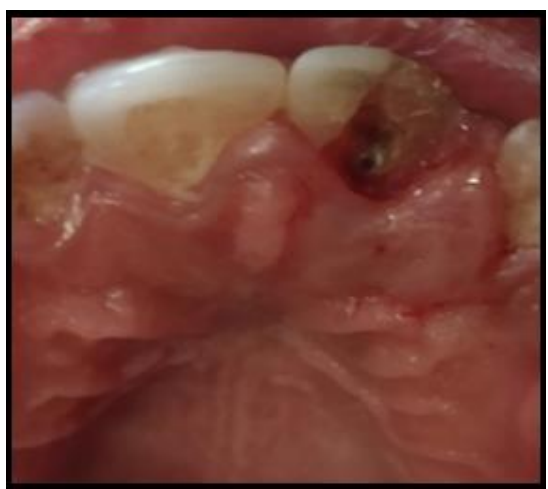

Fig. 8: Palatal aspect of tooth \#11 showing fracture margin at gingival level;

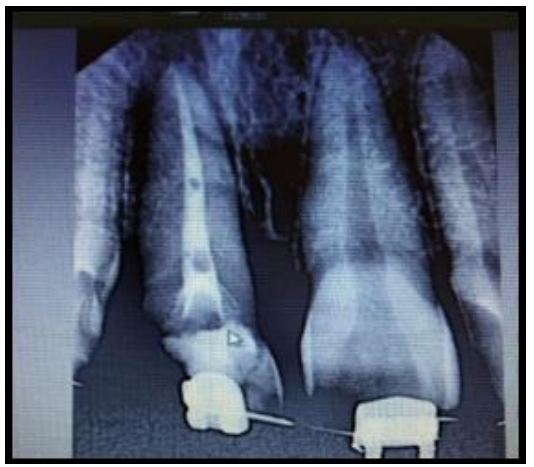

Fig. 9: Radiographic representation of extruded tooth \#11.

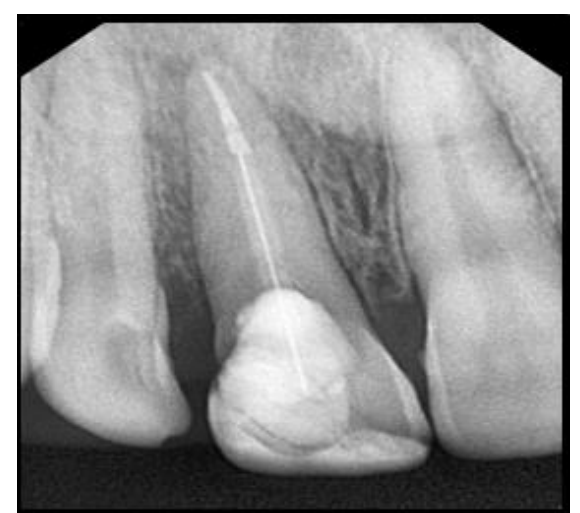

Fig. 10:Radiograph showing obturation followed by fiber post and core build-up of tooth \#11;

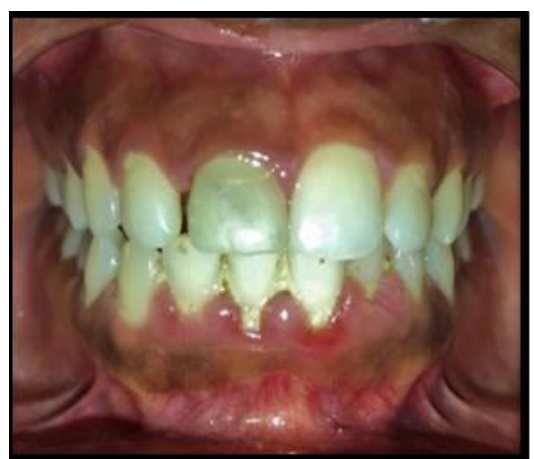

Fig. 11:Clinical picture showing uneven crown margins of tooth \#11 after extrusion;
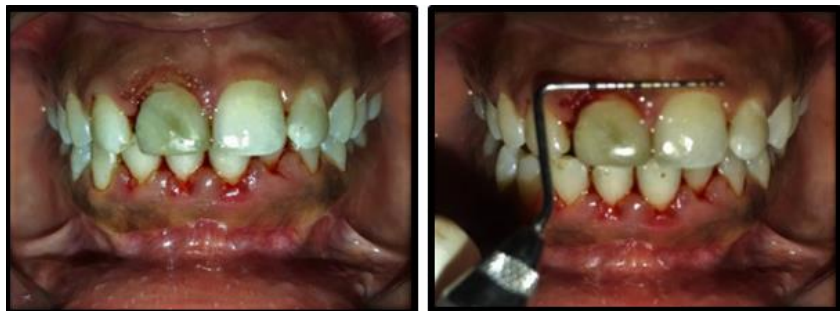

Fig. 12,13: Clinical picture post Laser assisted Crown lengthening to achieve proper contouring of tooth \#11;

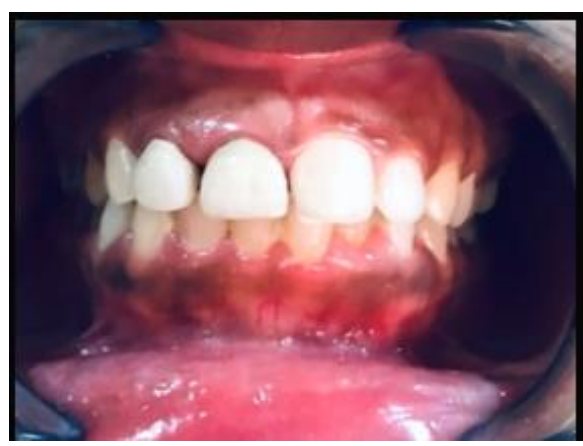

Fig. 14: Post operative clinical picture after PFM crown cementation of tooth \#11. 


\section{Discussion}

Dental trauma associated with complicated fracture in permanent anterior teeth, can be one of the most difficult situation in dental practice which demands a comprehensive treatment planning. Long term management of such cases can be complex due to uncertainties in prognosis and treatment time. ${ }^{12} \mathrm{~A}$ well-executed root canal treatment is the first step to control inflammation and pain management which builds a foundation on which any other treatment option will be based. ${ }^{13}$

This case report documents a subgingivally fractured permanent maxillary central incisor which is treated successfully with a multidisciplinary approach in cooperation with endodontist, orthodontist and prosthodontist.

Radiographic assessment showed the inadequate height of the remaining tooth structure to support a restoration. Hence, the options of surgical extrusion, forced orthodontic extrusion and periodontal surgery for crown lengthening were given a thought for consideration. Simple surgical crown lengthening would involve additional resection of the adjoining bone adjacent to the tooth that is to be lengthened, making it an invasive procedure at large, but, such osteotomy could be avoided by the use of orthodontic extrusion. ${ }^{14}$ Orthodontic extrusion is a biological way to expose sound tooth structure, which often requires prolonged treatment time. Rapid coronal movement with intense force provides faster clinical results, but has limited indications as it requires surgical correction following orthodontic treatment. ${ }^{15}$

According to Simon et al. orthodontic extrusion should become a routine procedure in dentistry. They also explained that the orthodontically extruded tooth must be stabilized for 8-12 weeks prior to fabrication of a permanent post and core. ${ }^{16}$ We have also found in the present study that 8-12weeks of stabilization of orthodontically extruded tooth has better prognosis.

Stern and Becker, in their study discussed orthodontic extrusion as an esthetic alternative to surgical crown lengthening and indicated that with an extrusive force, there was additional bone deposition lining the socket. ${ }^{17}$

At the end of the orthodontic extrusion procedure, there was some discrepancy between the gingival margin level of the treated tooth and the adjacent teeth which was not pleasant esthetically. Laser assisted periodontal surgery was performed for crown lengthening, but alone it was not enough to get appropriate results, and reduction of the residual bone with osteotomy could further reduce the bone support. $^{9}$

Due to inadequate remaining tooth structure, use of a glass fiber post and core was done for retention of a restoration. The use of fiber post will reduce the risk of root fracture as its inherent modulus of elasticity closely matches that of the dentin. ${ }^{18}$ For further reinforcement, considering cost factor and superior strength, metal ceramic crown was preferred for prosthetic rehabilitation.

Multidisciplinary approach is a key factor in successful functional and esthetic rehabilitation of a crown-root fracture. In this case, a subgingivally fractured right maxillary central incisor was treated endodontically, extruded orthodontically and restored with a fiber post and metal fused to ceramic crown.

\section{Conclusion}

Adjunctive orthodontic root extrusion is an essential clinical procedure that will enhance the integrated treatment planning process of tooth retention in endodonticorthodontic related cases. Being a conservative approach, extrusion of a fractured tooth has several advantages over extraction and prosthodontic replacement. It preserves the natural tooth and maintains the periodontal architecture. Long treatment duration is a disadvantage compared to extraction and replacement.

Careful evaluation of such complex cases is of utmost importance to achieve the best possible results which includes planned utilization of the multidisciplinary approach to focus on the treatment modality towards the positive outcome in terms of function and esthetics.

\section{Source of funding}

None.

\section{Conflict of interest}

None.

\section{References}

1. de Castro M.A.M.,Poi W R, de Castro J. C.M., "Crown and crown-root fractures: an evaluation of the treatment plans for management proposed by 154 specialists in restorative dentistry," Dent Traumatol 2010;26(3)236-42.

2. Castro JC, Poi WR, Manfrin TM, Zina LG. Analysis of the crown fractures and crown-root fractures due to dental trauma assisted by the Integrated Clinic from 1992 to 2002. Dent Traumatol2005;21:121-6.

3. Zerman N, Cavalleri G. Traumatic injuries to permanent incisors. Endod Dent Traumatol 1993;9:61-4.

4. Azami-Aghdash S, EbadifardAzar F, PournaghiAzar F, Rezapour A, Moradi-Joo M, Moosavi A, et al. Prevalence, etiology, and types of dental trauma in children and adolescents: systematic review and meta-analysis. Med J Islam Repub Iran 2015;29:234.

5. Enabulele JE, Oginni AO, Sede MA, Oginni FO. Pattern of traumatised anterior teeth among adult Nigerians and complications from late presentation. BMC Res Notes 2016;9:70.

6. Olsburgh S, JacobyT, Krejci I. Crown fractures in the permanent dentition: pulpal and restorative considerations. DentTraumatol 2002;18:103-115.

7. YoeliZ, Samet N, Miller V. Conservative approach to post traumatic treatment of maxillary anterior teeth: a clinical report.ProsthetDent 1997;78:123-6.

8. Villat C, Machtou P, Naulin-Ifi C. Multidisciplinary approach to the immediate esthetic repair and long term treatment of an oblique crown root fracture.Dent Traumatol2004;20:56-60.

9. Do_gu O” mürDede, Emine S, enTunc, AhmetUmutGüler, SebahatYazicio_glu. Multidisciplinary approach to a subgingivally fractured incisor tooth: A case report. J Dent SC2013;xx:1-5.

10. Bach N, Baylard J.F, Voyer R. Orthodontic extrusion: periodontal considerations and applications. J Can Dent Assoc 2004;70:775-80. 
11. Koyuturk A.E, Malkoc S. Orthodontic extrusion of subgingivally fractured incisor before restoration. A case report: 3-years follow-up. Dent Traumatol 2005;21:174-8.

12. Cheng L, Darendeliler MA, Vickers D. Orthodontics \& dental trauma. Australian society of orthodontics. Sydney: Surry Hills NSW 2010

13. Gluskin AH, Peters CI, Wong RDM, Ruddle CJ. Retreatment of non-healing endodontic therapy and management of mishaps. In: Ingle JI, Bakland LK, Baumgartner JC, Eds. Ingle'S Endodontics. 6th ed. Hamilton: BC Decker Inc. 2008;1088-161.

14. Rajan RR, Muddapp SC, RajendranP and Nair AS. Management of Cervically Fractured Central Incisors: A Multidisciplinary Approach. Open Dent J 2018;12:239-5.

15. MilardovicOrtolan S, Strujic M, Aurer A, Viskic J, Bergman L, Mehulic K et al. Esthetic rehabilitation of complicated crown fractures utilizing rapid orthodontic extrusion and two different restoration modalities. Int J ClinPediatr Dent 2012; 5(1):64-7.

16. Simon JHS, Kelly WH, Gordan DG, Ericksen GW. Extrusion of endontically treated teeth. JADA 1978;97:17-23.

17. Stern N, Becker A. Forced eruption: biological and clinical considerations. J Oral Rehabili 1980;7:395-402.

18. Lishen W, In Meei T, Marny Mohamed A, Abdullah D. An interdiscliplinary approach for management of an extensive carious premolar. Iran Endod J. 2018;13(3):403-6.

How to cite: Agarwal K, Samant PS, Shreya, Ashok N, Shyamal R. Multidisciplinary approach for management of traumatic subgingival crown-root fractured central incisor: A Case report. Indian J Conserv Endod 2020;5(1):22-6. 